\title{
AcMus: an Open, Integrated Platform for Room Acoustics Research
}

\author{
Marcelo Queiroz ${ }^{1}$, Fernando Iazzetta ${ }^{2}$, Fabio Kon ${ }^{1}$, Márcio Henrique A. Gomes ${ }^{3}$, \\ Fábio L. Figueiredo ${ }^{2}$, Bruno Masiero ${ }^{4}$, Leo K. Ueda ${ }^{1}$, \\ Luciana Dias $^{1}$, Mário Henrique C. Torres ${ }^{1}$ and Leandro F. Thomaz ${ }^{1}$ \\ ${ }^{1}$ Department of Computer Science \\ University of São Paulo \\ \{mqz I kon I lku | marioct I lfthomaz\}@ ime.usp.br \\ ${ }^{2}$ Department of Music \\ University of São Paulo \\ iazzetta@usp.br, fabioflf@hotmail.com \\ ${ }^{3}$ Centro Federal de Educao Tecnolgica de Santa Catarina - CEFETSC/Joinville \\ mavelar@cefetsc.edu.br. \\ ${ }^{4}$ Department of Electrical Engineering \\ University of São Paulo \\ bruno.masiero@poli.usp.br
}

Received 07 January 2008; accepted 04 July 2008

\begin{abstract}
This article describes the design, implementation, and experiences with AcMus, an open and integrated software platform for room acoustics research, which comprises tools for measurement, analysis, and simulation of rooms for music listening and production. Through use of affordable hardware, such as laptops, consumer audio interfaces and microphones, the software allows evaluation of relevant acoustical parameters with stable and consistent results, thus providing valuable information in the diagnosis of acoustical problems, as well as the possibility of simulating modifications in the room through analytical models. The system is open-source and based on a flexible and extensible Java plug-in framework, allowing for cross-platform portability, accessibility and experimentation, thus fostering collaboration of users, developers and researchers in the field of room acoustics.

Keywords: room acoustics, musical evaluation, acoustical parameters, impulse response measurement, computer simulation, optimization.
\end{abstract}

\section{INTRODUCTION}

Research in computational acoustics encompasses many disciplines, such as design and analysis of musical instruments, physical acoustics, room acoustics, and psychoacoustics. Each of these disciplines has its own specific theoretical background, methods, and goals.

In room acoustics, the project of recording studios, home theaters or movie theaters, and especially concert halls has received much attention from research groups and companies. Research focus has usually been placed on engineering and architectural issues rather than on music listening, and the use of commercial technology and methodology for such projects is still the rule. This is particularly true in developing countries, which depend on imported software, hardware, and qualified human resources.

Acoustical properties of rooms are specially important in the context of music reception and production. Some of these properties, referred to by Beranek [1] as "subjective attributes of acoustical and musical quality", such as 
presence, warmth, intimacy, clarity, diffusion, brightness and texture, are fundamental to any room with a musical use.

Several factors influence the quality of what we hear in a room. Controlling these factors is essential to determine what we hear in situations such as concerts, recordings, electroacoustic music performances and listening to pre-recorded material. D'Antonio [7] indicates as critical factors "the quality of the electronics, the quality and placement of loudspeakers, the hearing ability and placement of the listener, the room dimensions (or geometry, if non-cuboid) and the acoustical condition of the room's boundary surfaces and contents."

One goal of the AcMus project is to identify and analyze acoustical parameters that are relevant to rooms used for music listening and production, such as concert halls, lecture rooms, movie theaters and recording studios. These parameters allow for a quality assessment of such spaces and serve as guidelines in the correction of acoustic problems.

Another goal is to explore simulation techniques which allow the study of acoustic behavior of rooms during the design phase. This allows experimentation and evaluation of room designs, by modifying design parameters in simulation. When the design goals and constraints involve mathematically precise definitions, optimization techniques are available for the automatic search of optimal (or near-optimal) room designs.

The main goal of this article is to present new computational tools to evaluate and manipulate these acoustical parameters, through modern methodologies for software development, such as component-based design, used to build a flexible, extensible and adaptable system. It provides tools for measuring relevant acoustical parameters for rooms used for music listening and recording, and for simulating the acoustic behavior of such rooms, allowing experimentation with specific features such as geometry, acoustic absorption of walls and surfaces, and positioning of sound sources. It has an open-source implementation and is multi-platform, being freely available for use by architects, musicians and sound engineers, as well as for programmers interested in creating new components to extend its capabilities and customize it to different situations.

The article is organized as follows. Section 2 presents other works and software related to AcMus, and makes explicit the differences and contributions of the latter. Section 3 discusses the main modules of the AcMus integrated platform, namely the Measurement Module, the Simulation Module and the Utilities Module. Section 4 describes the AcMus interface, which is based on Eclipse, a flexible and extensible Java plug-in framework. Practical experience with the AcMus platform on concert halls in the state of So Paulo is reported on Section 5, and fur- ther works and conclusions are presented on Section 6.

\section{OVERView of Related Work}

There are many software packages for measuring impulse responses of rooms, calculating acoustical parameters, simulating the acoustics of room models and performing other tasks related to acoustical design and evaluation of rooms. Most of them are commercial packages designed for a single specific task and run on a single specific computer platform.

WinMLS (www.winmls.com) is a commercial package that runs on MS Windows. It implements techniques for measuring impulse responses (using as input signal swept sines, Maximum-Length Sequences or standard waveforms such as sinusoids, white noise or pink noise), from which it generates information such as Schroeder curve, Frequency Response and several acoustic parameters $^{1}$ (RT60, EDT, D50, C80, ST1, IACC, LF, G, among others).

ETF5 (www.etfacoustic.com) and Sample Champion (purebits.com) are other products for impulse response measurements and acoustical parameters evaluation that run on MS Windows and are similar to WinMLS.

Aurora [9] is a suite of freely available plug-ins for the Adobe Audition commercial package, that also runs on MS Windows. It is used for auralization of anechoic musical data using measured impulse responses, through headphones or on a dipole stereo setting (using crosscancellation techniques). It has a measurement module that uses a swept-sine technique for obtaining the binaural impulse response, and also post-processing tools for obtaining acoustical parameters such as RT, Tc, C80, STI, $\mathrm{LE}$ and LF, and also performing convolutions and inverse filtering.

Roomsim [5] is an implementation of the Image Source method for simulating impulse responses on cuboid rooms. This is a free, open-source implementation in MATLAB, which is a commercial package that runs on MS Windows, MacOS, Linux and Solaris. It uses frequency-dependent absorption coefficients in its simulation, tabulated for 24 building materials, and allows the simulation of mono and binaural impulse responses, as well as head-related effects on the impulse responses.

Several commercial packages for room simulations exist, and most use hybrid methods that combine Image Source with Ray-Tracing techniques. There are international competitions that explore how well these methods are able to simulate real rooms by comparing acoustical parameters obtained by measurements with ones obtained by simulations. Competitors of the 3rd International Round Robin on Room Acoustical Computer Simu-

\footnotetext{
${ }^{1}$ These parameters are defined by the ISO 3382 (1997) standard and will be examined in Section 3.
} 
lations were commercial packages such as CATT, dBRay, EPIDAURE, ODEON, RAMSETE, RAY, RAYNOISE and RAYPID, and other research software with no trade names and no available implementation [30, 4].

RoomOptimizer [7] is a commercial package for the optimal placement of sound sources and listeners in a cuboid room. The problem is defined by trying to obtain the flattest frequency response possible, by trying many positions of sources and listeners and recalculating the impulse response. It uses the Image Source method for simulating the impulse response, and Nelder \& Mead's Simplex heuristic for the optimization problem. It has presets for mono, stereo and 5.1 sound source configurations, but uses a single predefined average absorption coefficient for all surfaces.

The AcMus integrated platform has many common features with the above packages, and many differences as well.

AcMus provides both MLS and swept sine techniques for computing impulse responses of rooms, and allows computation of most relevant acoustical parameters, being similar to WinMLS, Aurora and ETF5 in this respect. But it includes another method for obtaining impulse responses based on a logarithmic sine sweep (Log-Sweep FFT).

Additionaly, AcMus is an open-source, fully integrated platform for room acoustic research. It runs on many platforms, including Linux, MacOS and MS Windows, being written in Java. It also has a graphical IDE that offers flexibility and extensibility through the Eclipse plug-in framework.

Furthermore, the AcMus research project has several independent applications that are scheduled for inclusion in the Eclipse interface environment.

It has simulation methods that employ not only the Image Source method (as Roomsim), but also a ray-tracing method that allows simulation of more complex rooms of polyhedral geometry.

It also has global optimization methods that allow optimal placement of sound sources and listeners (as RoomOptimizer), but also allows the search for optimal room designs, by varying room dimensions and acoustic materials of the surfaces.

Ultimately, the AcMus integrated platform will combine acoustic measuring tools with acoustic simulation and optimization tools in a single package for room acoustic research and design, allowing free flow of information between these modules.

\section{The AcMus Platform}

The AcMus Integrated Platform is composed of several modules and utilities designed for specific purposes. The main modules are the Measurement module, responsible for organizing measuring sessions and extracting rel- evant acoustical information from actual rooms, and the Simulation module, which aids in the acoustical design of new rooms or modification of existing ones. Other computational tools are grouped together in the Utilities module, which provides several independent tools related to room acoustics.

\subsection{Measurement Module}

Acoustical Parameters: All relevant physical acoustic parameters can be determined by the impulse response of the room. This response can be measured by several methods, the simplest of which is to generate a very short burst of sound energy, such as a gun shot or the explosion of a balloon, and recording the output. This is done in order to emulate a perfect impulse (or Dirac's Delta function). In the sequel the acoustical parameters computed by the AcMus platform are defined.

The most important acoustical measurement is $R e$ verberation Time or $R T 60$ [23, 1], which is related to the subjective impression of Liveness. This is a determining factor of the room quality and indicates how long it takes for a sound to decay $60 \mathrm{~dB}$ after the emission of the sound is interrupted. RT60 values are usually obtained for several frequency bands, defined by international standards (the AcMus software computes RT60 values for $63 \mathrm{~Hz}$, $125 \mathrm{~Hz}, 250 \mathrm{~Hz}, 500 \mathrm{~Hz}, 1 \mathrm{kHz}, 2 \mathrm{kHz}, 4 \mathrm{kHz}$ and $8 \mathrm{kHz})$. Other measures, such as RT30 or RT20 are used when the signal-to-noise ratio is poor and does not allow for direct measurement of RT60. It is also possible to calculate an expected, theoretical value of RT60, given by Sabine's equation [1], based on the room volume, area and absorption properties of the surfaces and area of the windows.

The Early Decay Time (EDT) represents the decay time for the first $10 \mathrm{~dB}$ of the initial reverberation curve. It is measured in octave frequency bands.

Definition (D50 or D80) is the ratio of the initial sound energy (corresponding to the first 50 or 80 miliseconds) to the total sound energy, which can be expressed as a function of the sound pressure $(p(t))$ as

$$
\frac{\int_{0}^{80 m s} p^{2}(t) d t}{\int_{0}^{\infty} p^{2}(t) d t} .
$$

Clarity (C50 or C80) is the logarithmic ratio of the energy within the first 50 miliseconds (for speech) or 80 miliseconds (for music) to the rest of the impulse response, or

$$
10 \log \left(\frac{\int_{0}^{80 m s} p^{2}(t) d t}{\int_{80 m s}^{\infty} p^{2}(t) d t}\right) .
$$

Clarity and Definition can be defined in terms of each other by the expression

$$
C_{\tau}=10 \log \left(\frac{D_{\tau}}{1-D_{\tau}}\right) .
$$


Sound Strength $(G)$ is related to the acoustic impression of sound level, and is defined by the logarithmic ratio of the sound pressure of the impulse response to the response of the same source placed 10 meters away in a free field (and, thus, no reverberation), given by

$$
\frac{\int_{0}^{\infty} p^{2}(t) d t}{\int_{0}^{\infty} p_{\text {free }}^{2}(t) d t} .
$$

Support Factor (ST1) is related to the subjective impression of Ensemble and is computed from a measurement taken $1 \mathrm{~m}$ away from a omnidirectional sound source on stage, by the expression

$$
10 \log \frac{\int_{20 m s}^{100 m s} p^{2}(t) d t}{\int_{0}^{10 m s} p^{2}(t) d t} .
$$

Central Time $(T s)$ is given by the expression

$$
T s=\frac{\int_{0}^{\infty} t \cdot p^{2}(t) d t}{\int_{0}^{\infty} p^{2}(t) d t}
$$

and corresponds to the gravity center of the whole impulse response, measured in miliseconds. A small value of Ts means that energy is concentrated at the beginning of the impulse response (which helps clarity), while a large value of Ts means that energy is distributed over time, giving the impression of a more reverberant space.

Also important are the Bass Ratio $(B R)$ and Treble Ratio $(T R)$, which are related to the subjective impressions of Warmth and Brilliance, and are defined as the relative contribution of respectively low and high frequencies to the overall spectral energy, and are computed from reverberation times in specific frequencies (denoted by $R T_{\text {freq }}$ ). The bass ratio is defined as

$$
B R=\frac{R T_{125}+R T_{250}}{R T_{500}+R T_{1000}},
$$

while the treble ratio is defined by

$$
T R=\frac{R T_{2000}+R T_{4000}}{R T_{500}+R T_{1000}} .
$$

The Ratio of Direct and Reverberant sound (RDR) is the logarithmic ratio of the energy of the direct sound to the energy of the rest of the impulse response, given by the expression

$$
10 \log \left(\frac{E_{d}}{E_{r}}\right) .
$$

The Inter-Aural Crosscorrelation Coefficient (IACC) is the maximum value for the correlation between signals arriving at the left and right ears, and is expressed in values ranging from -1 (left and right signals perfectly outof-phase) to +1 (identical signals).

Another parameter related to binaural listening is Lateral Fraction $(L F)$, given by the ratio between the energy coming from a lateral plane and the omnidirectional energy received by the same point, expressed as

$$
\frac{\int_{5 m s}^{80 m s} p_{\text {lat }}^{2}(t) d t}{\int_{0}^{80 m s} p_{\text {omi }}^{2}(t) d t} .
$$

Both IACC and LF together are related to the subjective notion of Spatial Impression.

The Initial Time Delay Gap (ITDG) is related to the subjective impression of Intimacy and corresponds to the time interval between the arrival of the direct sound and the first reflection, and is a decisive factor for the acoustic quality of an environment. It relates to the subjective feeling of intimacy. This is of particular importance for small rooms in which reflective surfaces are too close to the listener, thus making the reflected sound reach the listener immediately after the direct sound. To avoid extremely small values of the ITDG, recording studios use design concepts such as LEDE (Live End Dead End) [8] or RFZ (Reflection-Free Zone) [16]. In the LEDE concept half the room is designed to avoid primary reflections and the other half is designed to enhance diffusion. Rooms based on the Reflection-Free Zone create a spatial zone surrounding the listener which is free of early reflections.

Computing the Impulse Response: Accurate methods for measuring the impulse response consist in generating a reference signal in the room, such as a noise or a sine sweep, measuring the effect of the room on the original signal, and applying spectral methods to recover the impulse response. These methods offer a much better signal-to-noise ratio and allow for repeatability of tests, since they use predefined excitation signals. Two of these methods have been implemented in AcMus: the MLS (Maximum Length Sequency) and a newer method called LSF (Log Sweep FFT), that is based on TDS (Time Delay Spectrometry) [12].

The effect of a room on sound can usually be seen as a linear time-invariant system, characterized by its impulse response $h(t)$. The Fourier Transform of the impulse response is called the Transfer Function, or Frequency Response of the room, and is a function $H(\omega)$ of frequency. The action of the room on a input signal $x(t)$ is expressed in the time-domain as a convolution

$$
y(t)=(x * h)(t)=\int_{-\infty}^{\infty} x(s) h(s-t) d s,
$$

which corresponds, in the frequency-domain, to a multiplication of the corresponding spectra:

$$
Y(\omega)=X(\omega) H(\omega) .
$$

The MLS method [25, 27] is largely used in acoustic measurement systems, and uses a signal based on a periodic sequence of pseudo-randomly distributed positive and negative impulses. The MLS signal sounds like 
white noise, and shares with it the property of having a flat energy distribution in the frequency domain. The computation of the impulse response by the MLS method takes advantage of this fact and the following properties:

- The cross-correlation of input and output of a linear system is equivalent to the convolution of the impulse response with the auto-correlation of the input. In symbols:

$$
R_{x y}(t)=\left(h * R_{x x}\right)(t) .
$$

- The auto-correlation function of a single MLS sequence of length $\mathrm{N}$ approaches a Dirac's Delta function as $\mathrm{N}$ increases: if $x(t)$ is a MLS sequence of length large enough, then $R_{x x}(t) \approx \delta(t)$.

- Dirac's Delta is neutral with respect to convolutions, i.e., $(x * \delta)(t)=x(t)$ for any signal $x(t)$.

From these properties it is immediate that the impulse response $h(t)$ can be approximated by the crosscorrelation of a MLS signal $x(t)$ with the recorded output of the room $y(t)$ :

$$
h(t) \approx R_{x y}(t)=\int_{0}^{\infty} x(s) y(t+s) d s .
$$

A similar argument applies to periodic MLS sequences, as usually done in practice. The computation of the impulse response by this process uses a Fast Hadamard Transform, which optimizes the processing of matrices generated by cross-correlation.

Another approach to measure an impulse response is the Time Delay Spectrometry (TDS), which consists in aplying a sinusoidal signal with linearly varying frequency, and recording the output [12]. The Log Sweep FFT Method (LSF) $[9,18]$ is an evolution of the TDS that uses a logarithmic sine sweep as excitation signal. It increases the signal-to-noise ratio for lower frequencies because its spectrum is analogous to pink noise, as opposed to the almost white noise spectrum of the linear sweep (TDS), and thus gives more time for lower frequencies to build up and for the room to respond to them.

If $X(\omega)$ is the pink spectrum of the LSF signal and $Y(\omega)$ is the spectrum of the recorded signal, the transfer function of the room can be obtained by

$$
H(\omega)=\frac{Y(\omega)}{X(\omega)},
$$

and the impulse response by the inverse FFT of $H(\omega)$.

This method has the advantage of allowing the elimination of the influence of the loudspeaker: if $L(\omega)$ is the spectrum of the loudspeaker, obtained by measurements in free field (or in an anechoic chamber), the composite

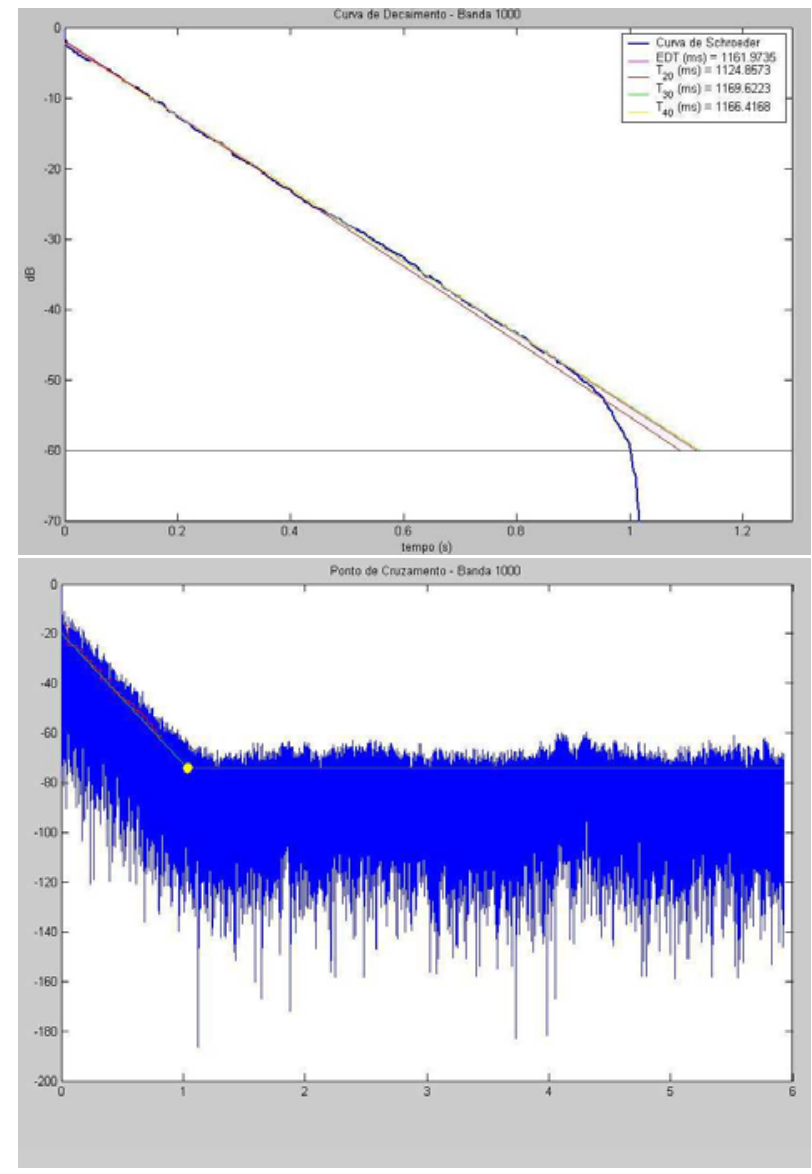

Figure 1. Schrder integration and respective $\mathrm{dB}$ decay curve.

system loudspeaker+room acts according to the following equation:

$$
Y(\omega)=X(\omega)[L(\omega) H(\omega)],
$$

and so the transfer function can be easily obtained by

$$
H(\omega)=\frac{Y(\omega)}{X(\omega) L(\omega)} .
$$

Tests performed with the AcMus platform indicate that LSF has a better signal-to-noise ratio, being more recommended than MLS for low cost systems subject to non-linear responses and critical signal-to-noise ratio.

After the impulse response is computed, the energy decay curve is obtained by the Schrder Integration method (see Figure 1)

$$
E(t)=\int_{t}^{\infty} h^{2}(s) d s=\int_{0}^{\infty} h^{2}(s) d s-\int_{0}^{t} h^{2}(s) d s
$$

and from this curve all of the mentioned acoustic parameters can be directly obtained. 


\subsection{Simulation Module}

A traditional way of obtaining acoustical parameters of a room is making measurements on-site. When this is not possible, one may build a miniature replica (scale model) and make measurements in the model to obtain approximate values for the real parameters (in the real room). Nowadays, computer models are very popular, and many acoustic consultants use them together with scale models during the design of a new room.

One major concern in the AcMus project is to provide robust models and prediction tools that may be used in simulation without need of directly measuring acoustic data in a real room or a scale model. This is clearly a necessary condition for the automatic search for an optimal room design, since a huge number of different design parameters need to be tested, including the position of sound sources and listeners, specific materials for the acoustic treatment of the surfaces, and ultimately even the dimensions or the geometry of the room.

Although the reliability of computer simulations is not completely demonstrated, there are precise conditions under which several algorithms are reported to give reliable results for the computation of acoustic parameters, such as reverberation time, early decay time, clarity, definition, lateral fraction and interaural cross correlation coefficient [10].

The simulation model implements geometrical acoustics and optimization techniques that allow the assessment of acoustical parameters of room designs and the solution of design problems, such as the optimal placement of sound sources and listeners, and optimal choices of geometry and surface materials to achieve desired acoustic properties, such as prescribed reverberation times for specific frequency ranges, or minimal harmonic distortion (used in the sense of timbral coloring, as in [7]). For spectral properties such as the latter it is desirable to obtain approximations of the frequency response of the room.

Acoustic phenomena such as modal coupling, comb filtering or speaker-boundary interference influence the frequency response of a room, determining amplification or damping of specific frequencies or frequency bands. First, the presented geometrical techniques are discussed for the special case of specular reflection; later, the effect of diffusion is considered (see also [1,2]).

Modal coupling is a result of the interaction of the sound source and the reflective surfaces of a room. It is associated with the constructive or destructive interferences in sound waves traveling in different directions. Closed specular-reflection paths (loops) have a direct impact on wavelengths that are submultiples of the path length. This interference affects mainly those listening positions lying within the closed paths, though diffusion may blur this effect to adjacent positions. According to how many surfaces the corresponding closed path hits, room modes are

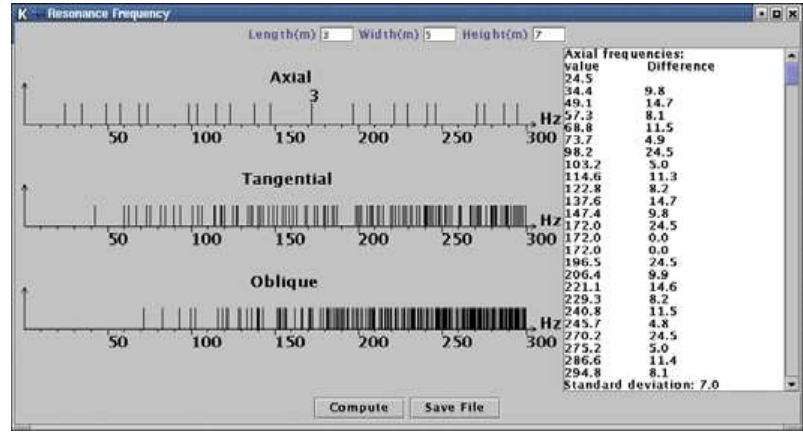

Figure 2. Modal response of a cuboid room.

called axial, tangential and oblique, and these can be computed through the formula

$$
f\left(n_{x}, n_{y}, n_{z}\right)=\frac{c}{2} \sqrt{\left(\frac{n_{x}}{L_{x}}\right)^{2}+\left(\frac{n_{y}}{L_{y}}\right)^{2}+\left(\frac{n_{z}}{L_{z}}\right)^{2}},
$$

where $f$ is the frequency, $c$ is the speed of sound, $\left(L_{x}, L_{y}, L_{z}\right)$ are the room dimensions and $\left(n_{x}, n_{y}, n_{z}\right)$ are integers related to the closed path (see Figure 2). One way to approximate the effect of room modes for particular source and listener positions is to generate paths leaving the source in the directions $\left(n_{x} L_{x}, n_{y} L_{y}, n_{z} L_{z}\right)$, and for each path that comes sufficiently close to the listener, calculate the corresponding frequencies and amplification or damping factors.

The term comb filtering is applied to a particular type of interference corresponding to the superposition of the direct sound and the first reflection on a nearby wall, e.g. a wall right behind a loudspeaker, determining a spatial pattern of amplification or damping for each particular frequency and listener position. The distance from the listener to a nearby wall also determines a comb filtering effect due to the interference of the direct sound and the sound reflected on the wall behind his/her head.

More generally speaking, it is relatively easy to consider all early reflections that reach the listener from a particular sound source. This done by geometrical acoustic methods that identify sound paths in a room. These paths may include those interference paths responsible for modal coupling and comb filtering effects, but also more general sound paths.

Geometrical Acoustics: Two popular methods for the acoustical simulation of sound reflections based on geometrical models are ray-tracing and virtual image source $[29,7,22]$.

Ray-tracing is a technique also used in Computer Graphics that substitutes the traditional sound propagation of spherical (or planar) wavefronts by linear rays of sound. Each ray leaves the source and follows a linear 


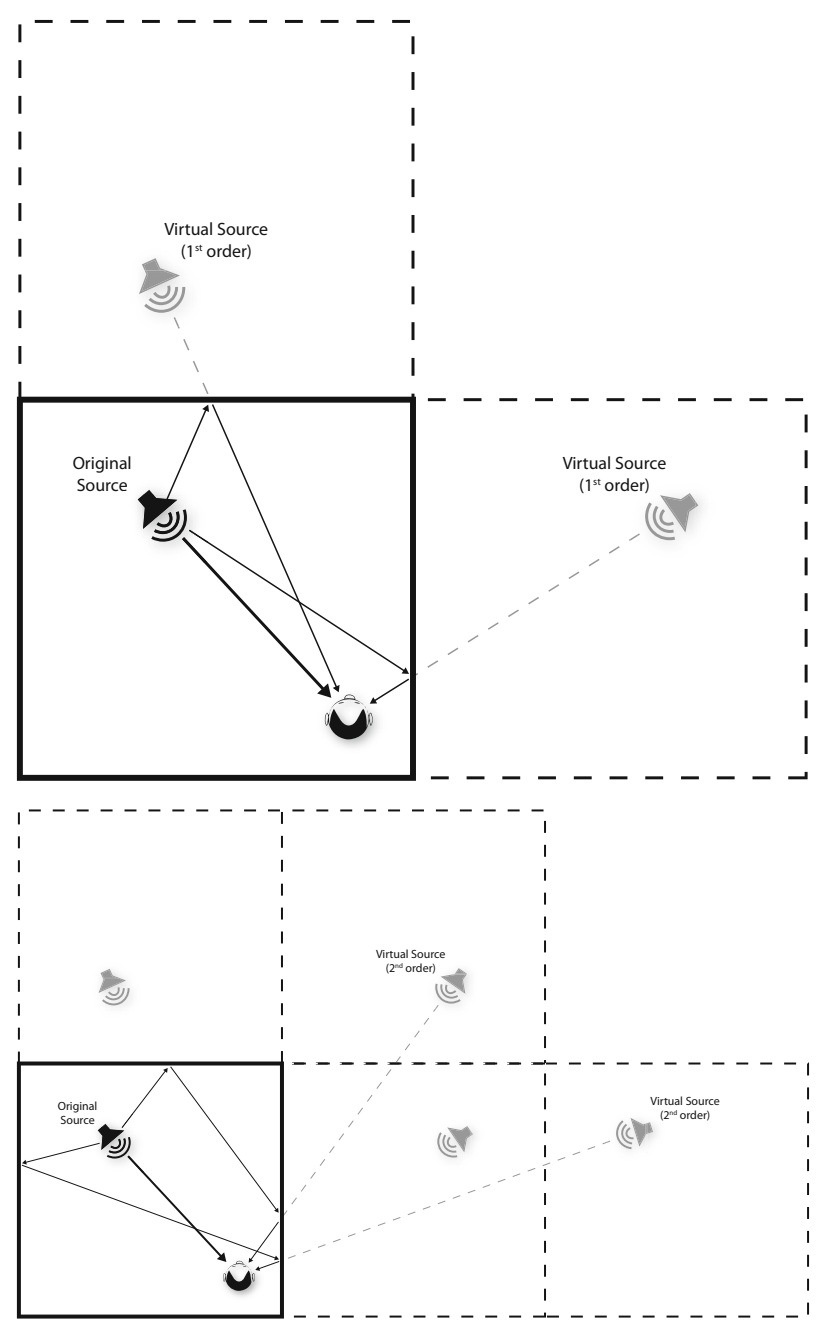

Figure 3. First and second order virtual image sources and corresponding paths inside the real room.

path, suffering a specular reflection at each wall it traverses. By applying damping factors to the energy of the ray at each reflection (given by the absorption coefficient of the wall), and also considering the absorption of the air (related to the square of the distance), one can build an echogram, which is a map of all reflections, along with corresponding delays and energy values. This echogram is related to the impulse response of the room, and can be used to obtain the acoustical parameters or a proper impulse response and its corresponding frequency response [17].

According to the image source model, a sound ray that is reflected by a wall is identical to a direct ray coming from a virtual source, located on the other side of the wall, at the same position as the specular image of the original source, as in Figure 3.

One can think of a free field with many virtual image sources (specular reflections of the original source), that irradiate the same signal in a synchronized fashion. The simulation can be performed by tracing direct paths from the virtual sound sources to the listener, and multiplying the response factor by the reflection coefficient of each wall that the path traverses.

This approach is closely related to the ray-tracing method, with the difference that by using image sources fewer rays are actually generated that never reach the listener. Both techniques can also be combined in a hybrid ray-tracing/virtual image method, by using image sources for early reflections (with increased accuracy) and raytracing for later reflections (with improved computational performance) [29].

Calculating the position of the images is trivial in the case of cuboid rooms, but is computationally expensive for complex geometries, especially if the walls are highly reflective. In this case, the number of images increases exponentially with the number of reflections. This restricts the application of the virtual image method to home theaters, small recording studios and concert rooms with very simple geometries.

Note that both virtual image source and ray-tracing techniques do not deal directly with modal effects, which may limit their application to higher frequencies or large rooms. On the other hand, responses obtained with the image sources method may help identify comb filtering problems $[29,7]$.

The techniques above provide a simulated impulse response that allows computation of the acoustic parameters with the same methods mentioned in the previous section. It also allows the experimentation of alternative room designs, by changing the description of the room, including its geometry, acoustic properties of the surfaces, position of sound sources and listeners, etc.

Optimization: By establishing specific design goals it is possible to automatically search for optimal design parameters, for instance using optimization techniques $[31,7,20]$. At each step in the search algorithm, a new impulse response is obtained by geometrical acoustic techniques and then the acoustic parameters and the frequency response can be computed.

The main goal in the optimization module is to minimize the harmonic distortion of the room, which implies timbral coloring and might be undesirable under critical listening conditions. The main design variables considered here are the room dimensions and reflection coefficients of the surfaces (walls, floor, ceiling), where ranges for possible room dimensions, reflection coefficients and positions of sound sources and listeners are given.

One of many possible design criteria for the minimization of the harmonic distortion is trying to obtain a nearly flat frequency response, where "nearly flat" presupposes the minimization of some measure of deviation from flat- 
ness, such as the standard deviation or least-squares errors of the frequency response [7]. An alternative is asking for a frequency response that resembles as close as possible a given ideal frequency response, not necessarily flat. Another alternative is trying to match as close as possible the reverberation times for specific frequency ranges to a pre-established reverberation curve.

All these criteria correspond to minimizing the deviation of a function obtained by simulation from a given ideal function, thus defining analogous optimization problems that can be solved with the same techniques.

The overall optimization goal may also be stated in various forms. One of them is the problem of optimally placing sound sources and listeners in specific positions in such a way that harmonic distortion is minimized. Another possibility is to determine room attributes, such as geometry or acoustical properties of the surfaces, so that harmonic distortion is improved in several listening positions.

Suppose that the range of possible source positions is described by a set $\mathrm{S}$ (stage) and the range of possible listener positions is described by a set A (audience). Let $\mathrm{D}$ describe the set of feasible room attributes (including geometry and surface absorption coefficients).

To optimize the harmonic distortion in the average case (i.e. in an average source-listener pair) the harmonic distortion must be integrated over all possible $s \in S$ and $l \in A$.

Another possibility is to optimize the worst-case scenario, i.e., trying to guarantee that the worst possible combination of source and listener positions will have a minimal harmonic distortion. This is achieved by minimizing the supremum of the harmonic distortion over all possible $s \in S$ and $l \in A$.

Figure 4 shows typical graphs of the harmonic distortion as a function of listening positions. Values of deviation from flatness are shown on a horizontal plane situated at the ear level of a seated listener. It is easy to see that function values have few regions of relative flatness (sweet spots), displaying wide variations on most of the room.

In any case all problems have the following properties: lack of closed formulas and convexity-like properties for the objective functions and mixing of continuous and discrete decision variables that lead to a combinatorial behavior or the problem. The algorithms used are global optimization heuristics that combine stochastic methods with local optimization procedures. This approach has great relevance since the objective functions involved have too many local minima with values that are very far from the true optimal value.

Two optimization algorithms have been implemented: the Density Clustering method [26] and a Grid-based local search [19].
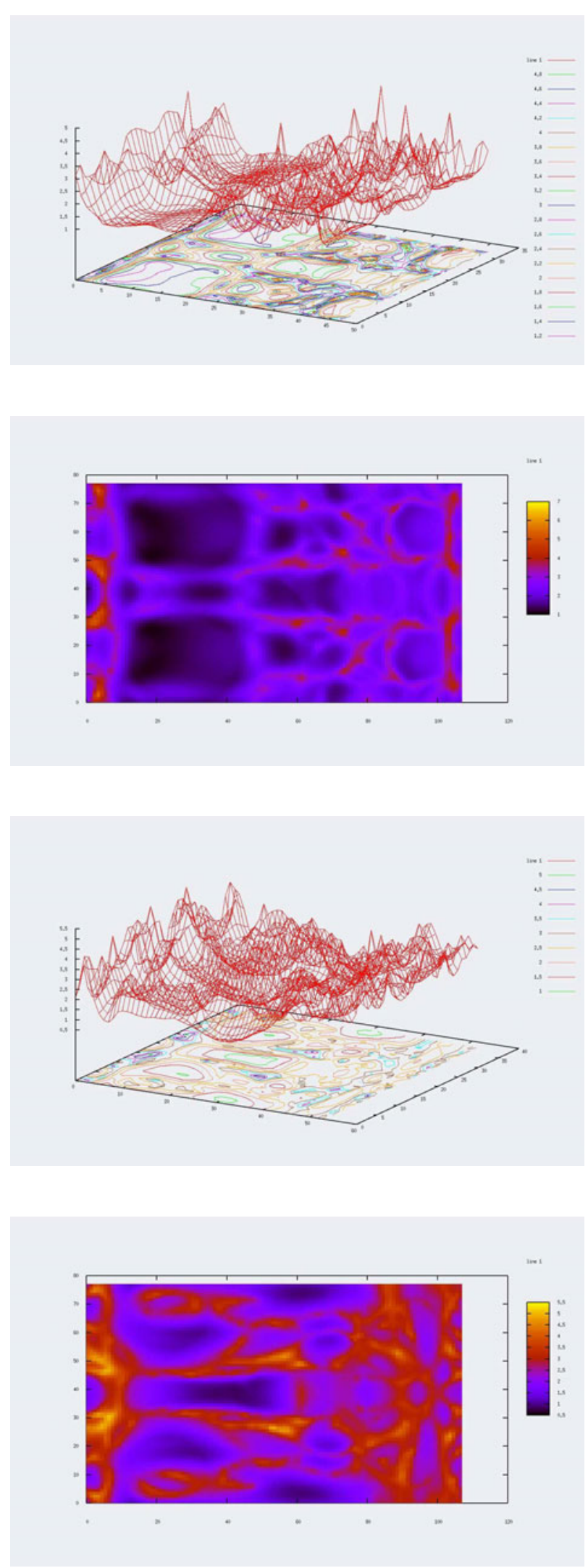

Figure 4. Harmonic distortion on a horizontal plane for two rooms. Each pair of graphs represents different views of the same functions. 
Density Clustering is a stochastic optimization heuristic that has two phases: the global phase generates random feasible points using a uniform distribution on the feasible set, and evaluates the objective function on these points; the local phase applies a local optimization procedure on a subset of the feasible points, corresponding to those with better objective function values. This generates a set of local minima, around which clusters are defined, according to critical distance criteria. These clusters save computation on future rounds of the global phase, by discarding points that lie in the neighborhood of a previously known local minimum.

The local optimization procedure used in the local phase in the current implementation is the Grid-Based search. This is a deterministic method that looks for local minimizers on a discrete grid of feasible points, by moving through adjacent points with decreasing function values until a minimum (on the grid) is found. The outer iteration is responsible for subdividing the grid, thus allowing refinement of the solution and guaranteeing convergence of the method.

Computational tests have been conducted for the optimal placement of sources and listener in idealized rooms, and compared to similar tests made with the Room Optimizer [7]. Although the results are preliminary [20], it has been verified that the Grid method obtains consistently better points than the Simplex method used in Room Optimizer. The Density Clustering heuristic tends to refine the solution of the Grid search by finding many local minima and selecting the best of them. Although it takes more time, it increases the probability of finding better local minima, or eventually the true global optimum.

The bottleneck of the optimization procedure still is the acoustic simulation, since every evaluation of the objective function requires its own simulated impulse response. To avoid repeating costly simulations and save computation, the Grid method collects all known points where previous simulations have been performed, together with their objective function values. The practical impact of this strategy is nevertheless very limited, specially for the Density Clustering method, which usually has to evaluate hundreds of different designs for each problem.

\subsection{Utilities Module}

This module offers functions for specific tasks relating to acoustic measurement and simulation or the acoustical design of rooms, as well as functions for generating many types of audio signals, filtering and spectral analysis. Many tools have been implemented as independent Java applications and are gradually being included in the AcMus integrated platform.

For instance, Figure 5 shows a module for obtaining reverberation times of a room, given the room dimensions and a description of the materials of the surfaces. Another module computes the modal response of a cuboid room based on the same data, and was shown on Figure 2.

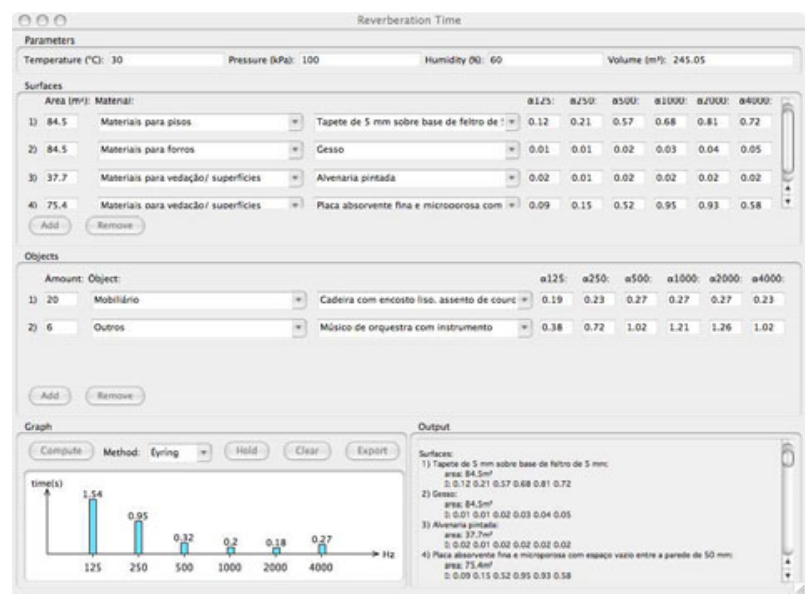

Figure 5. Reverberation times of a room.

There is also a graphical tool that allows comparison of two waveforms, and a convolution tool that may be used for auralization. Other specific modules follow:

Calculation of Sound Propagation Speed: the speed of sound is affected by several environmental factors, such as relative humidity and temperature. This module implements a simple formula for obtaining the speed of sound as a function of these values.

Design of Schröder diffusers: The resonance modes for lower frequencies (up to $300 \mathrm{~Hz}$ ) are important for the acoustical characterization of a room. On the other hand, the diffusion and reflection patterns are of greater importance for medium and high frequencies [21]. Ideally, the overall acoustic quality of a room increases with the homogeneity of diffusion factors. In order to solve the problem of lack of reflection from side walls in concert rooms, Manfred Schröder designed a highly diffusing surface [24] that can be used in critical hearing conditions, such as concert rooms and recording studios. It consists of evenly spaced wells of different depths, that depend on the frequency range that the diffuser is supposed to affect.

Helmholtz absorbers: the control of low frequencies within a room based solely on the treatment of surfaces or the geometry is particularly difficult. For specific low frequencies it is recommended to use tuned absorbers to avoid prominent resonances below $200 \mathrm{~Hz}$. Helmholtz absorbers are well-suited to this task, and can be designed with this tool.

\section{IMPLEMENTATION}

The development of the software was guided by some specific concerns, discussed in detail in [15]. Since it 
is intended to promote exchange of results with other research groups, the software is based on open source codes, and in the same spirit, whenever possible open source codes and algorithms from other research groups are also used. This relates to the requirement that the system be platform independent, and for this reason it has been mostly developed in Java, running easily on Linux, MacOS and MS Windows.

The system is organized in modules, that are integrated in a way that allows seamless flow of data, regardless of the variety of data types and structures involved in the system.

The implementation is based on the sophisticated Eclipse platform (www.eclipse.org), which is a powerful, generic, extensible, open set of computer tools for developing programs. It has the same basic purpose of any other IDE (Integrated Development Environment), such as Visual Studio, Delphi or NetBeans, but goes beyond by providing a foundation for constructing and running contributed plug-ins written in Java, allowing it to be largely extended. Plug-ins are pieces of programs with a defined structure that add functionality to the platform, and may define extension points to let other plug-ins extend it. Almost the entire platform is built upon this model, even its main subsystems, resulting in a highly extensible system.

The AcMus software is implemented as a set of Java plug-ins integrated into the Eclipse platform, which facilitates the system's extensibility and flexibility, enabling the construction of an open platform for acoustics experimentation that is freely available to researchers in the field.

Figure 6 displays the audio player, which shows a logarithmic sine sweep signal used for measurements. The upper part shows the audio signal, and the lower part shows the time-varying spectrum of this signal.

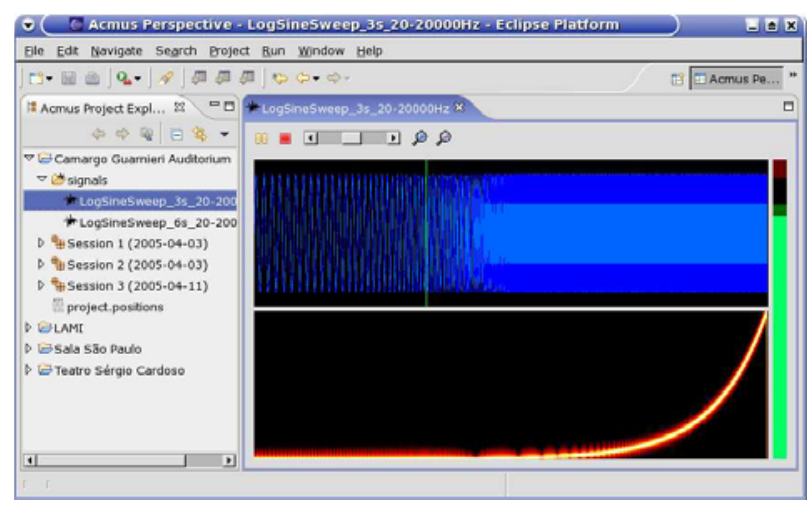

Figure 6. Audio player showing a logarithmic sine sweep.

In a measurement, a microphone and a loudspeaker, representing a listener and a sound source, are placed at chosen positions inside a room. The software then feeds the loudspeaker with a signal and at the same time the microphone captures the room's response to this sound. After that, the software processes this recording to calculate parameters that describe acoustic characteristics of the room for that particular position of listener and source. Usually these measurements are taken in a large number of positions and repeated several times. A major concern of the implementation effort has been the quality of the user interface, trying to lessen the burden of taking countless measurements in a room.

The Measurement module takes advantage of the Eclipse Resources Plug-in to let the user store measurements taken from different rooms in hierarchical folders (Figure 7). The Project folder represents a room (the

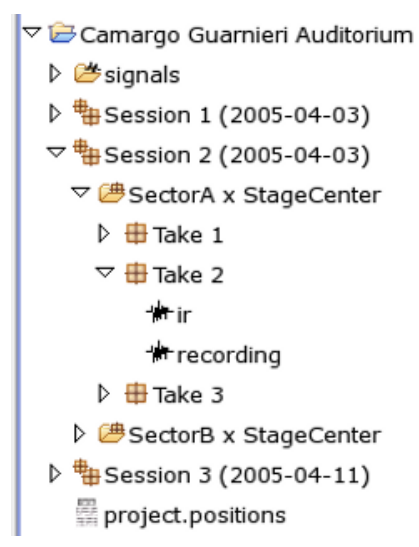

Figure 7. Hierarchical organization of folders in Measurements module.

Carmargo Guarnieri Auditorium in the example). The 费 Session folder groups measurements taken in the same period (in the example there are two sessions on april 3rd and another session on april 11th 2005). The 曲 Set folder stores repeated measurements on the same spot (e.g. SectorA x StageCenter in the Figure), which improves reliability of the results. The $\$$ Measurement folder stores the audio file of the room's impulse response for each individual take and the output of the response analysis.

The Measurement Module provides wizards for the creation of each kind of folders. The user may input additional information about the new folder such as date, time, equipment, comments, and so on. Figure 8 depicts the wizard for the creation of a Session folder.

Inside each project folder there is a file called project.positions, that keeps a list of microphone and loudspeaker positions in a graphical representation of the floor plan of the room (Figure 9), which provides documentation and helps manage the measurements.

The Input Signal Manager allows creation and storage of reference signals used for measuring the impulse re- 


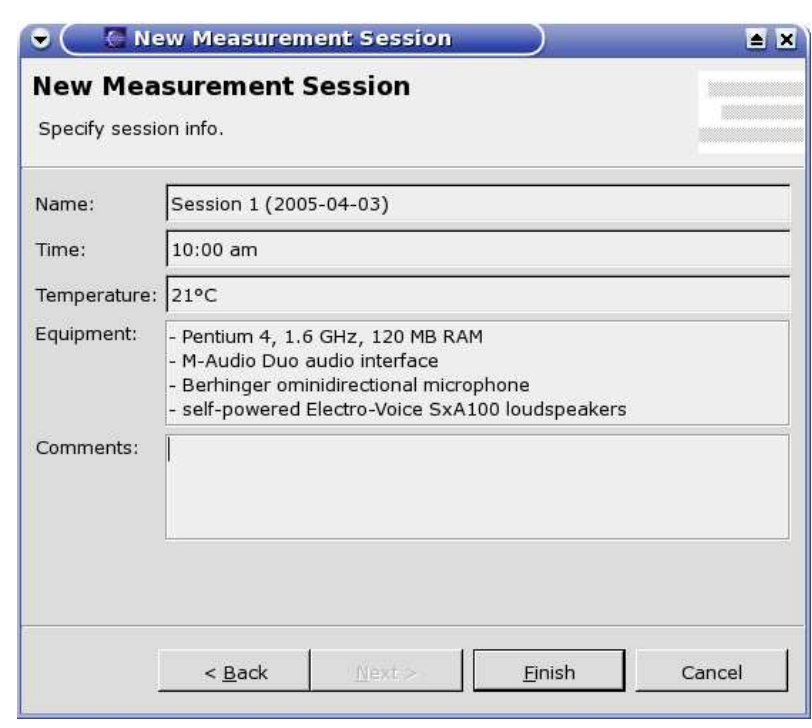

Figure 8. The Session creation wizard.

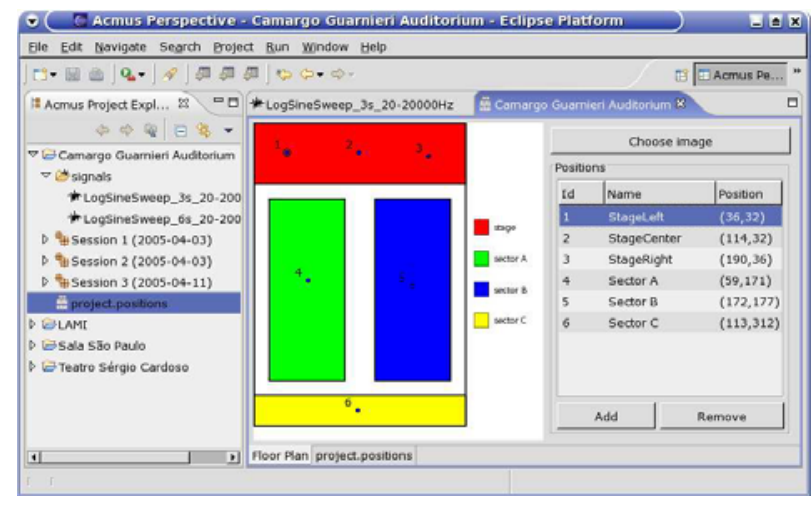

Figure 9. Graphical representation of the project.positions file.

sponse, in the signals folder. The software provides a wizard (Figure 10) that creates logarithmic sine sweeps from parameters given by the user, such as initial and ending frequencies and total duration of the signal. A user may also use any other audio file as input signal, keeping in mind that it directly affects the quality of the impulse response obtained.

The Measurement interface (Figure 11) displays the chosen input signal and allows the recording of the room response to that signal. The software also records the signal sent to the loudspeakers, which is taken as a reference to sync the signal that is captured by the microphones. The "Calculate IR" button performs a spectral processing of both recorded signals, producing the impulse response of the room (Figure 12), for that measurement.

Before calculating the acoustic parameters from the impulse response, the software offers the possibility of

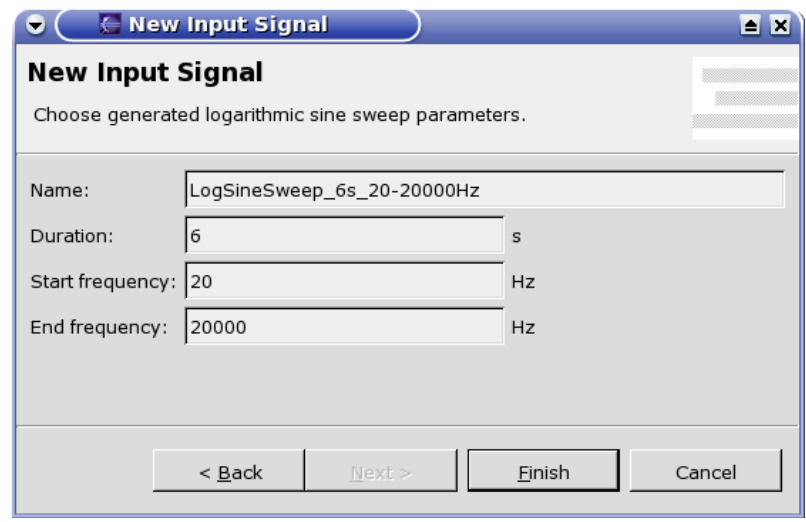

Figure 10. The Signal wizard.

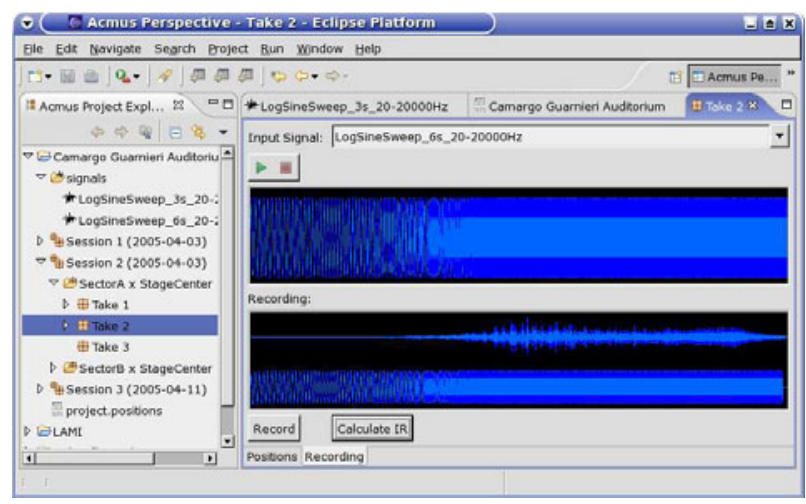

Figure 11. Measurement interface displaying the original signal on the upper part and both recorded signals on the lower part.

applying some treatment to the signal, such as the methods of Lundeby [28], Chu [6] and Hirata [13]. After this treatment, the software calculates the following acoustic parameters: reverberation time (RT), early decay time (EDT), sound strength $(\mathrm{G})$, clarity (C), definition (D), center time (Ts), bass ratio (BR) and treble ratio (TR), as shown in Figure 13.

\section{Practical Studies}

Among the goals of the practical studies is the investigation of the relation between objective acoustic parameters that can be determined by measurements and specific calculations, and subjective acoustic parameters, as determined by individuals' sensitivity and perception in specific listening and performing environments [14].

Terms that are commonly used in the evaluation of concert halls include liveness, warmth, brilliance, intimacy, clarity, definition and spatial impression, and have been studied in comparison with the objective, measurable parameters by Beranek [1].

The present comparison has been conducted by analyzing, with the AcMus platform, the acoustics of concert 


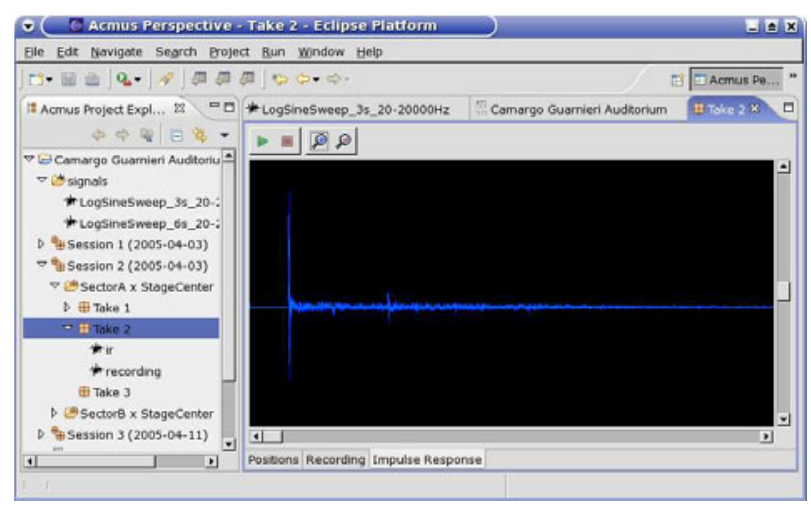

Figure 12. Impulse response obtained by spectral processing of the recorded signal.

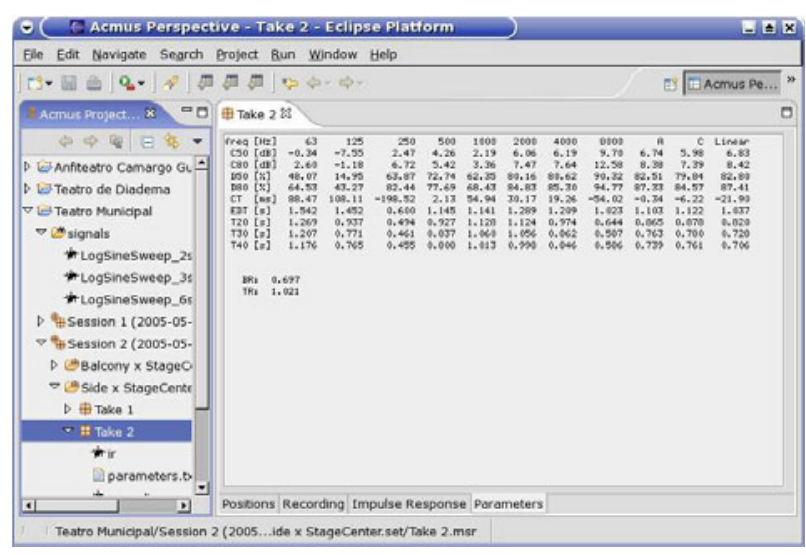

Figure 13. Acoustic parameters obtained from an impulse response.

halls in the state of São Paulo that carry regular performances of chamber and symphonic ensembles, and relating the measurements to subjective evaluations made by professional musicians according to their psychoacoustic sensitivity.

The following system was used for the tests: a laptop PC computer (Pentium 4, 1.6GHz, 120MB RAM), M-Audio Duo and Edirol FA-101 digital audio interfaces, Berhinger ECM 8000 and Neumann U-87 omnidirectional microphones and self-powered Electro-Voice Sx200a loudspeakers.

Measurements were carried out in several concert halls in the city of So Paulo (Anfiteatro Camargo Guarnieri, Teatro de Diadema, Teatro do Memorial da Amrica Latina, Theatro Municipal de So Paulo, Teatro So Pedro and Teatro Srgio Cardoso). Three of them will be discussed in the sequel: Theatro Municipal de So Paulo, which hosts the Orquestra Sinfnica Municipal, Teatro So Pedro, former headquarter of OSESP and Anfiteatro Camargo Guarnieri, which hosts the University's Symphony Orquestra (OSUSP).

These halls have different architectural and acoustical characteristics. The Theatro Municipal holds 1500 people in a Italian-style shaped audience, has a significant part of the floors covered by carpets, offers 4 floors for the audience and has therefore a very high ceiling. Teatro So Pedro holds about 700 people in a shell-shaped audience, and its surfaces are mainly of masonry. Anfiteatro Camargo Guarnieri also holds about 700 people, but its shape is rectangular and its walls are covered with wood.

Figure 14 shows impulse responses taken in different positions in these three theaters. Patterns change considerably for different positions in the same theater. As a rule, stage measurements produce short and dry impulse responses as opposed to measurements made in the center of the audience. This is explained respectively by the directivity of the source and the higher amount of reflection.

Many measures were taken for each position, and parameters were calculated in two ways: taking the average of each parameter for every impulse response, and calculating the parameters of an averaged impulse response. Figure 15 shows a comparison of values for Clarity (C80) on the main floor of Anfiteatro Camargo Guarnieri computed with both methods. The maximum standard deviation was $4.2 \%$ for frequencies above $125 \mathrm{~Hz}$.

The MLS and LSF methods have presented very similar impulse responses and gave practically the same parameter values. A typical comparison for these differences can be observed in Figure 16, comparing RT60 values obtained with the two methods in a stage position in Anfiteatro Camargo Guarnieri.

Since this analysis aims at investigating rooms designed for musical performance, it was taken into consideration the directionality of sound emission observed in musical instruments. Measurements were carried out using a directional source to stimulate the room, and results were compared to measurements done with omnidirectional sources to investigate the impact of this factor.

Reverberation times for all theaters can be seen in Figure 17. It can be seen that Theatro Municipal and Teatro So Pedro presented high reverberation times in the low frequency range, which is due to the high ceiling in the first case and the masonry walls in the second, as opposed to the Anfiteatro Camargo Guarnieri with its wooden walls that help diffusion. For higher frequencies these values tend to converge to a single value.

Directly related to the previous observation are the values for Clarity (Figure 18), which are higher in Anfiteatro Camargo Guarnieri than in the other two halls. When comparing these values in the center and the back of the audience, it is noticeable that the Anfiteatro Camargo Guarnieri presents greater Clarity in the center than in the back seats, for the whole frequency range. The reason for this is the fact that this hall does not have balconies, and so its back seats are part of the main floor, as 

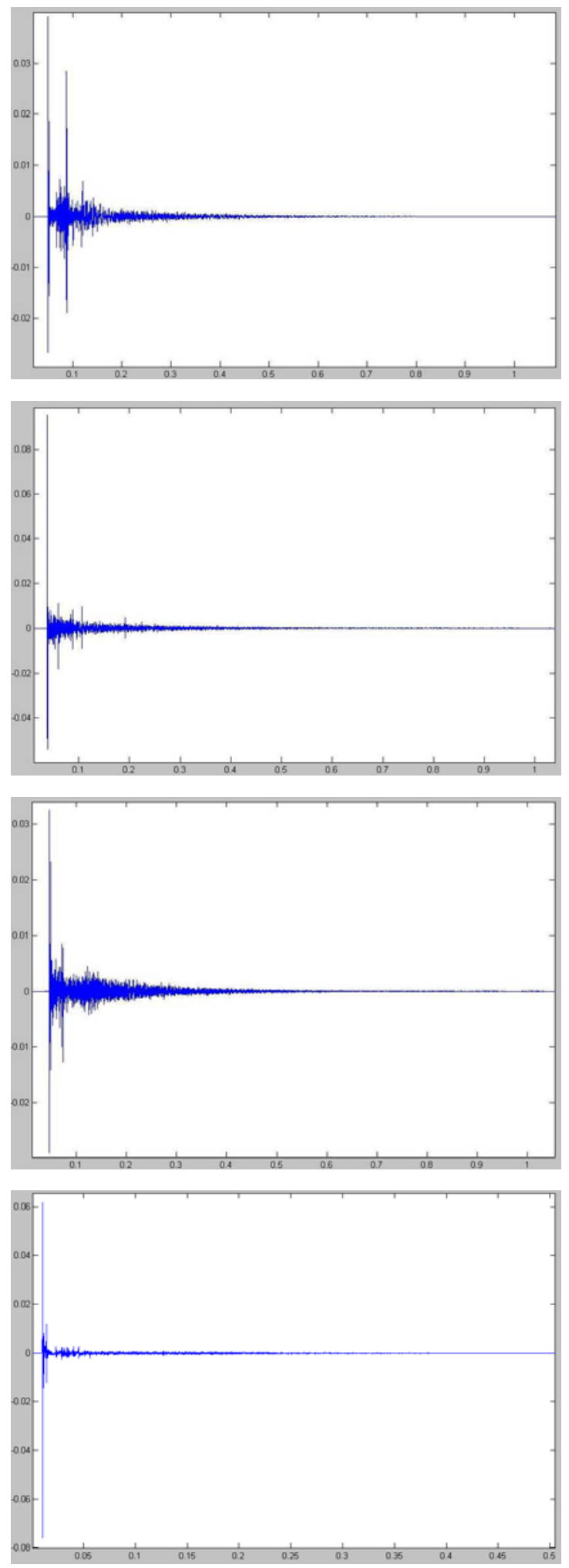

Figure 14. Some measured impulse responses: (a) lateral main floor and (b) central main floor in Theatro Municipal, (c) stage in Anfiteatro Camargo Guarnieri and (d) balcony of Teatro So Pedro

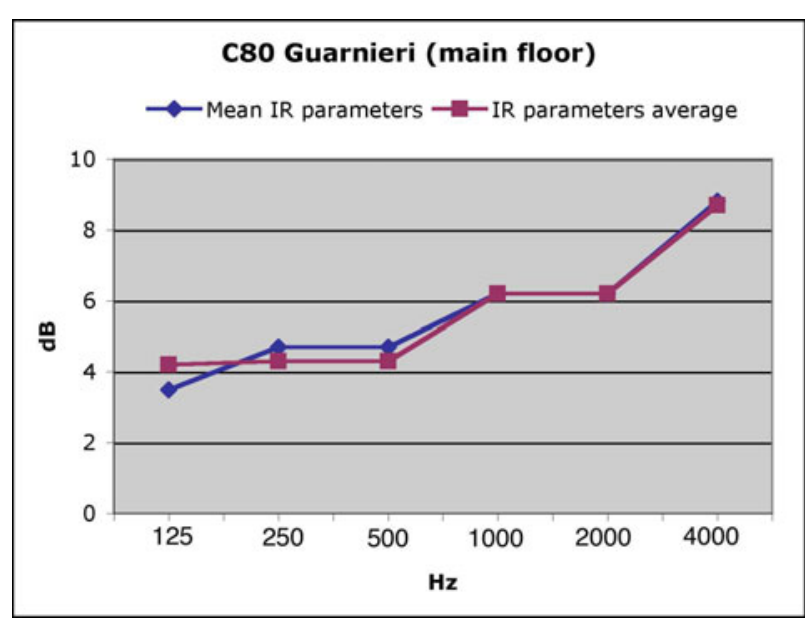

Figure 15. Averages of IRs versus averages of parameters.

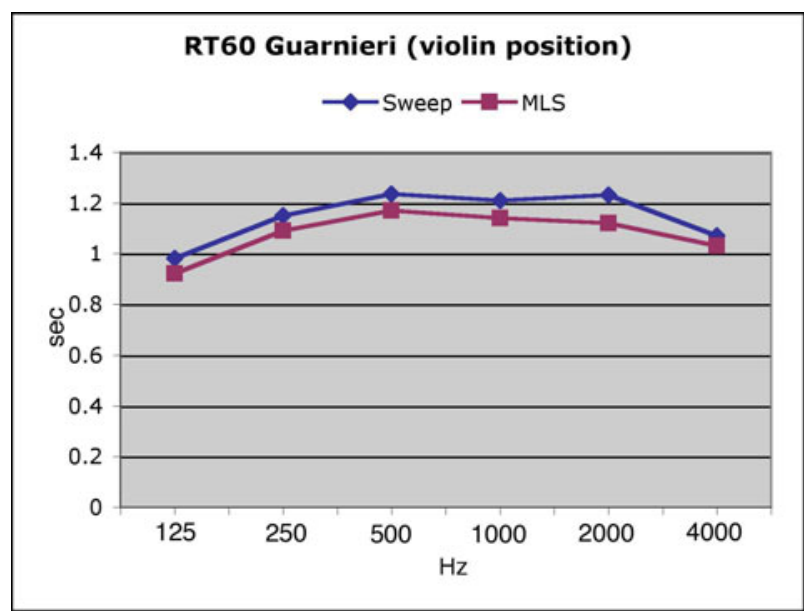

Figure 16. RT60 values obtained with MLS and LSF signals.

opposed to the two other halls. In Theatro Municipal and Teatro So Pedro the balconies act as barriers for reflections coming from above [3], which explains their higher values of Clarity.

With respect to frequency ratios (Figure 19), the Anfiteatro Camargo Guarnieri proved to be more balanced, whereas the large carpet surfaces in Theatro Municipal collaborate in the reduction of brightness.

The main parameter affected by the direction of the source is Clarity, a problem which was already detected in measurements of lecture halls [11]. For low frequencies the behaviour of loudspeakers is almost omnidirectional, acquiring directionality as a function of frequency. This explains the high Clarity values in high frequencies, whose behavior is less diffuse (more specular) and thus carries less reverberant energy. RT60 values presented less sensitivity to directivity of the source. 

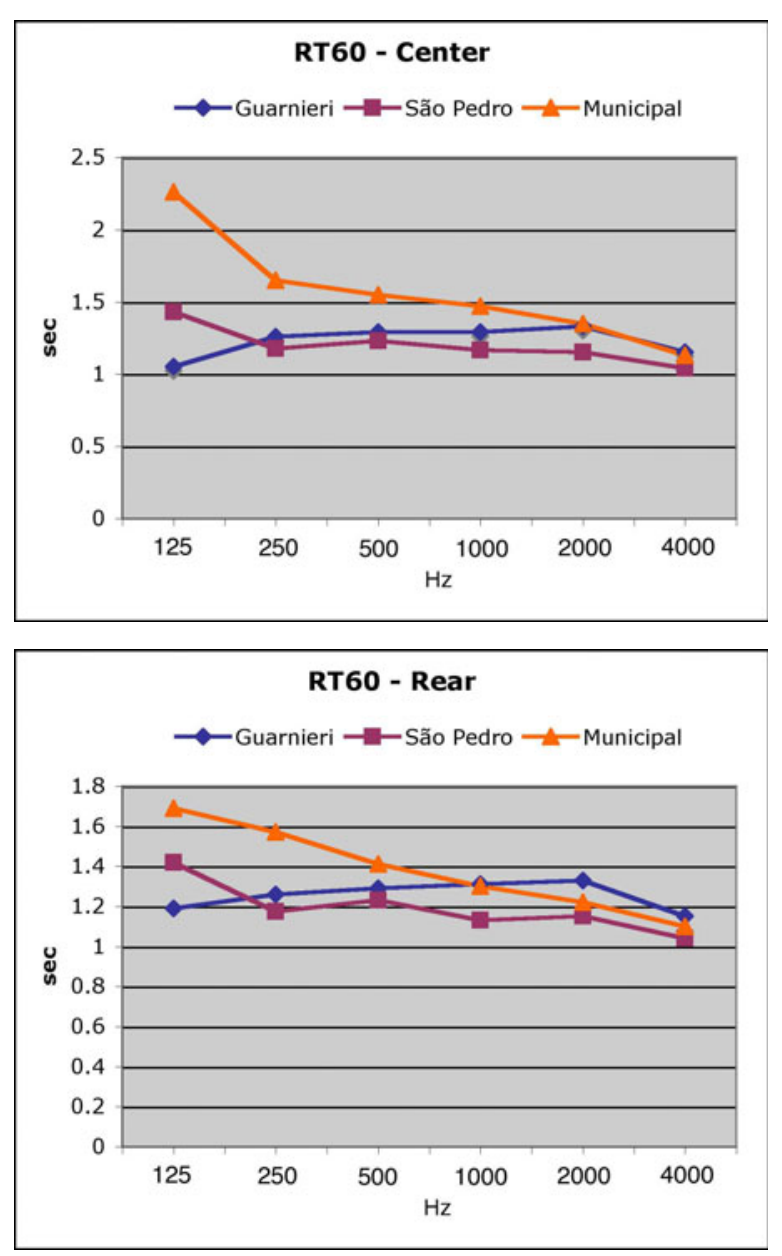

Figure 17. Reverberation times on the center and back of the audience.

Clarity and Definition on the stage tend to have larger values than on the audience. This is a desirable characteristic both for the conductor as well as for musicians playing in chamber ensembles. ITDG values are lower in the stage (average of $4 \mathrm{~ms}$ ) than in the audience (average of $13 \mathrm{~ms}$ ). In the back of the audience the impulse responses indicate a higher diffusion and lower values for the direct/reverberant energy ratio.

The Ratio of Direct to Reverberant sound energy (RDR) was computed using musical samples instead of reference signals. This allowed psychoacoustical comparisons of the same musical piece heard on different parts of a theater. RDR values are regarded as correlated to the subjective impression of intimacy [14]. It can be seen from the measurements (Figure 20) of several theaters that average RDR values become smaller as a function of distance from the source, ranging between $80 \%$ and $90 \%$ on the stage, $60 \%$ and $70 \%$ at the front seats, and floating around $30 \%$ at the back seats, with few exceptions. It is noticeable that RT60 values do not follow the same pat-
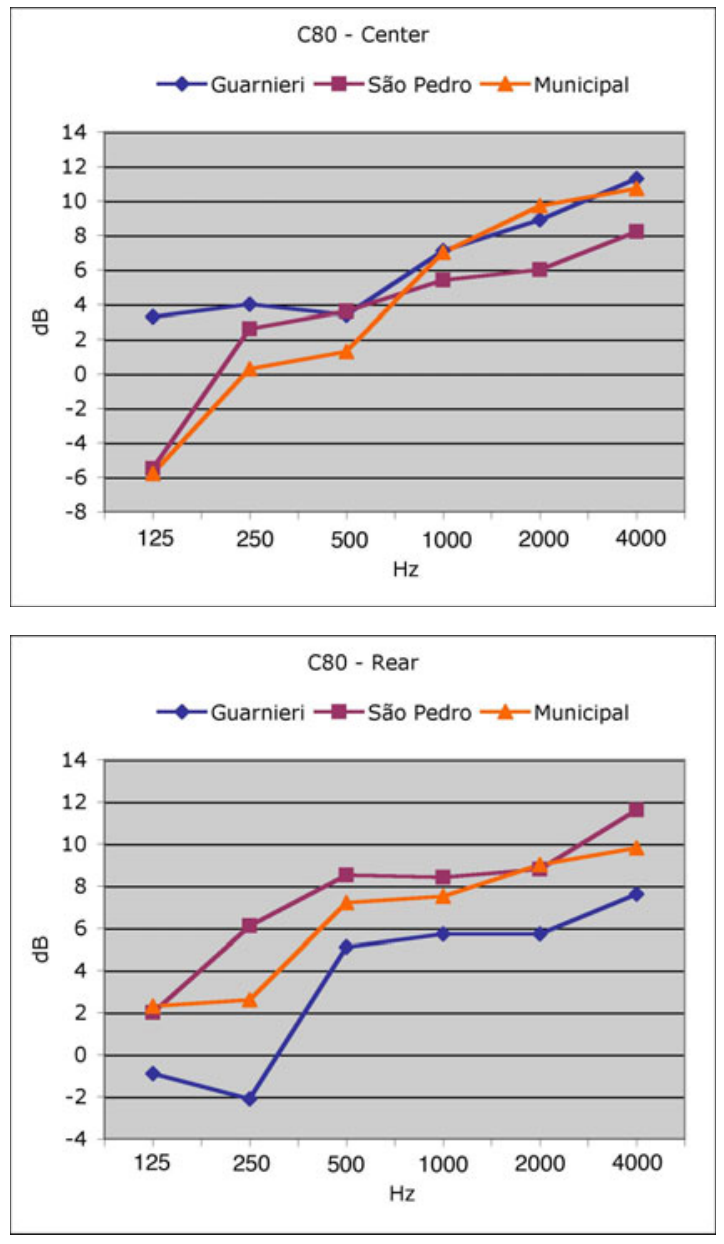

Figure 18. Clarity (C80) values on the center and back of the audience.

tern, suggesting that RDR values also play a role in the subjective perception of reverberation.

Table 1 summarizes the data collected on these theaters, and allow cross-comparisons of previous figures. Each column describes a different theater, showing their values for the C80, RT60, BR/TR and RDR parameters. The first section of the table gives general information about each theater. The next section corresponds to parameters obtained at a central seat, and the last section to a back seat at the audience of the theaters. The table shows frequency-dependent values for three representative frequencies $(250 \mathrm{~Hz}, 1000 \mathrm{~Hz}$ and $4000 \mathrm{~Hz})$.

\section{CONClusions AND Further Work}

This article presented the AcMus integrated platform for Room Acoustics experimentation and research. It achieved its primary goals of producing high-quality software for measurement of acoustic parameters relevant for music listening and production, fostered interdisciplinary 


\begin{tabular}{|c|c|c|c|c|}
\hline & & Theatro Municipal & Teatro So Pedro & Anfiteatro Camargo Guarnieri \\
\hline \multicolumn{5}{|c|}{ Characteristics } \\
\hline & seats & 1500 & 636 & 360 \\
\hline & sions (m) & $23.00 \times 24.00$ & $9.60 \times 15.50$ & $21.41 \times 28.30$ \\
\hline & surfaces & carpet & masonry & wood \\
\hline \multicolumn{5}{|c|}{ Parameters (center) } \\
\hline \multirow[t]{3}{*}{$\mathrm{C} 80(\mathrm{~dB})$} & $250 \mathrm{~Hz}$ & 0.290 & 2.570 & 4.000 \\
\hline & $1000 \mathrm{~Hz}$ & 6.950 & 5.430 & 7.140 \\
\hline & $4000 \mathrm{~Hz}$ & 10.670 & 8.250 & 11.240 \\
\hline \multirow[t]{3}{*}{ RT60 (s) } & $250 \mathrm{~Hz}$ & 1.646 & 1.183 & 1.256 \\
\hline & $1000 \mathrm{~Hz}$ & 1.463 & 1.171 & 1.293 \\
\hline & $4000 \mathrm{~Hz}$ & 1.122 & 1.037 & 1.159 \\
\hline BR & & 1.341 & 1.060 & 0.890 \\
\hline $\mathrm{TR}$ & & 0.793 & 0.862 & 0.931 \\
\hline RDR (fron & & $81.087 \%$ & $55.217 \%$ & $61.087 \%$ \\
\hline \multicolumn{5}{|c|}{ Parameters (rear) } \\
\hline \multirow[t]{3}{*}{$\mathrm{C} 80(\mathrm{~dB})$} & $250 \mathrm{~Hz}$ & 2.538 & 6.118 & -2.115 \\
\hline & $1000 \mathrm{~Hz}$ & 7.451 & 8.423 & 5.725 \\
\hline & $4000 \mathrm{~Hz}$ & 9.769 & 11.615 & 7.577 \\
\hline \multirow[t]{3}{*}{ RT60 (s) } & $250 \mathrm{~Hz}$ & 1.565 & 1.174 & 1.261 \\
\hline & $1000 \mathrm{~Hz}$ & 1.291 & 1.132 & 1.313 \\
\hline & $4000 \mathrm{~Hz}$ & 1.094 & 1.038 & 1.153 \\
\hline BR & & 1.119 & 1.175 & 0.924 \\
\hline TR & & 0.862 & 0.952 & 0.972 \\
\hline RDR & & $29.565 \%$ & $25.870 \%$ & $16.596 \%$ \\
\hline
\end{tabular}

Table 1. Summary of measurements for Theatro Municipal de So Paulo, Teatro So Pedro, and Anfiteatro Camargo Guarnieri

research and brought together researchers with complementary backgrounds from several research institutes.

The AcMus project is work-in-progress, and hopefully for a long time: though much has been achieved during its first 5 years, many ideas and sub-projects are being developed at the time of writing or planned for future implementation.

The measurement module is by far the most advanced one, having been tested and used by other researchers not involved with the AcMus project.

The simulation module is still somewhat scattered in many independent applications, with no integrated interface built into the Eclipse plug-in environment. The code of these applications is being translated at the time of writing and will be available soon.

Many improvements are under study for this module. For instance, absorption coefficients for different acoustic materials are available as functions of frequency, but as a rule geometrical models use mean values of these parameters. The consideration of a more realistic model of absorption makes the implementation considerably more complex, but the advantages for enhancing the simulation for frequency-dependent processing (such as estimating the harmonic distortion) are clear. The absorption coefficient also varies with the incidence angle of a sound ray, and that could be taken into account.

Other factors that might improve the quality of simulated impulse responses is the treatment of sound diffusion and diffraction. Each natural property of sound propagation increases realism of the simulation at the expense of ever-increasing computational complexity. Part of the job of designing any simulator is to analyze the trade-off between quality and time, which for acoustic purposes always involves the question of what exists in theory versus what is really felt subjectively (psychoacoustically).

An implementation of a ray-tracing technique for polyhedral geometries is under current development. It will allow a more thorough comparison of simulated impulse responses with measured ones for concert rooms, such as the ones here presented, which do not have cuboid geometries.

With good simulated impulse responses it is possible to build an auralization module for assessing the acoustic quality of rooms with musical experiments. Some pre- 


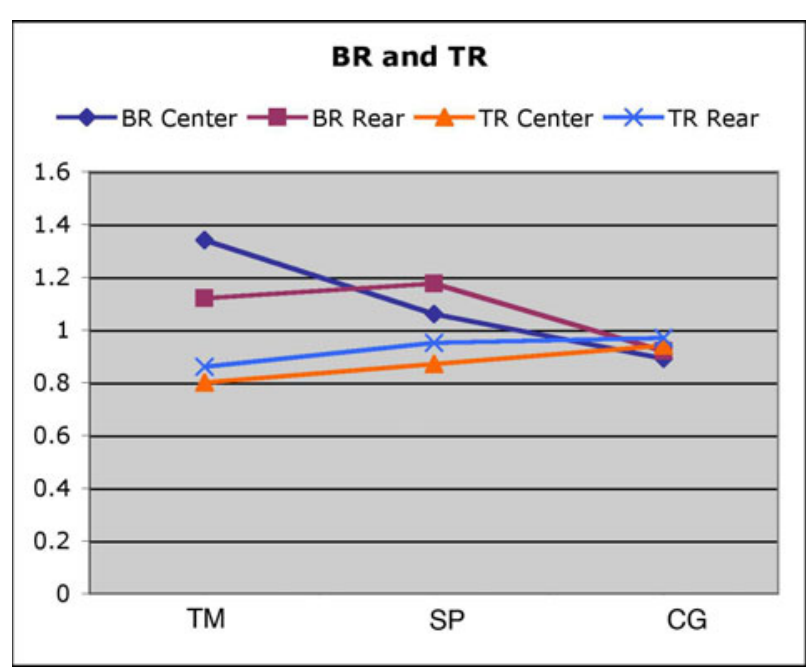

Figure 19. Bass and Treble Ratios.

processing of the impulse responses obtained by geometrical methods is necessary to guarantee that auralized signals will not sound sterile, artificial or have artifacts (i.e. that they will be psychoacoustically credible).

\section{ACKNOWLEDGMENTS}

This work has been supported by CNPq and FAPESP grant 02/02678-0.

\section{REFERENCES}

[1] L. L. Beranek. Music, Acoustics and Architecture. John Wiley and Sons, 1962.

[2] L. L. Beranek. Concert hall acoustics - 1992. Journal of the Acoustical Society of America, 92(1):139, 1992.

[3] L. L. Beranek. Concert Halls and Opera Houses: Music, Acoustics and Architecture. Springer Verlag, 2004.

[4] I. Bork. Report on the 3rd round robin on room acoustical computer simulation - part i: Measurements. Acta Acustica united with Acustica, 91:740752, 2005.

[5] D. Campbell; K. Palomäki; G. Brown. A matlab simulation of 'shoebox' room acoustics for use in research and teaching. Computing and Information Systems Journal, 9(3):48-51, 2005.

[6] W. T. Chu. Comparison of reverberation measurements using schröder's impulse method and decaycurve averaging method. Journal of the Acoustical Society of America, 63(5):1444-1450, 1978.

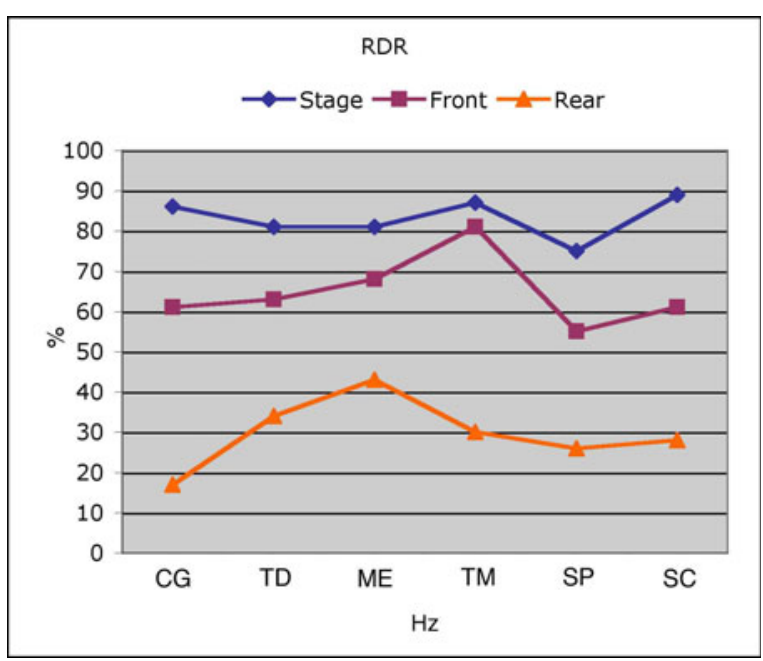

Figure 20. RDR values for stage, center and rear audience in several theaters.

[7] P. D'Antonio; T. J. Cox. Room optimizer: A computer program to optimize the placement of listener, loudspeakers, acoustical surface treatment and room dimensions in critical listening rooms. In Proceedings of the 103rd Convention of the Audio Engineering Society, Preprint 4555, Paper H-6, 1997.

[8] D. Davis; C. Davis. The LEDE concept for the control of acoustic and psychoacoustic parameters in control rooms. Journal of the Audio Engineering Society, 28(9):585-595, 1980.

[9] A. Farina. Simultaneous measurements of impulse response and distortion with a swept sine technique. In Proceedings of the 108th AES Conference, France, 2000.

[10] M. H. A. Gomes; M. Vorländer; S. N. Y. Gerges. Measurement and use of scattering coefficients in room acoustic computer simulations. In Proceedings of the Acustica 2004, 2004.

[11] F. Arruda; R. Guedes. A influência da direcionalidade de fontes sonoras na avaliação experimental da qualidade acústica de salas. In Proceedings of the XIX Meeting of the Brazilian Society of Acoustics, 2000.

[12] R. C. Heyser. Acoustical measurements by time delay spectrometry. Journal of the Audio Engineering Society, 1967.

[13] Y. Hirata. A method of eliminating noise in power responses. Journal of Sound and Vibration, 82:593$595,1982$. 
[14] F. Figueiredo; F. Iazzetta. Parâmetros acústicos em salas de música: análise de resultados e novas interpretações. In Anais do 4.o Congresso de Engenharia de Áudio. São Paulo: Audio Engineering Society Seção Brasil, pages 66-71, 2006.

[15] L. K. Ueda; F. L. Figueiredo; F. Iazzetta; F. Kon. A user-friendly graphical system for room acoustics measurement and analysis. In Anais do 10.o Simpósio Brasileiro de Computação Musical. Belo Horizonte: Sociedade Brasileira de Computação, pages 118-129, 2005.

[16] P. D'Antonio; J. H. Konnert. The RZF/RPG approach to control room monitoring. In Proceedings of the 76th Convention of the Audio Engineering Society, Preprint 2157, Paper I-6, 1984.

[17] H. Kuttruff. Room Acoustics. Elsevier Applied Science, 1991.

[18] S. Müller; P. Massarani. Transfer function measurements with sweeps. Journal of the Audio Engineering Society, 49:443, 2001.

[19] I. D. Coope; C. J. Price. On the convergence of grid-based methods for unconstrained optimization. SIAM Journal on Optimization, 11(4):859869, 2001.

[20] L. Dias; M. Queiroz. Localização de fontes e ouvintes em salas de escuta. In Anais do 10.o Simpósio Brasileiro de Computação Musical. Belo Horizonte: Sociedade Brasileira de Computação, pages 130-141, 2005.

[21] J. H. Rindel. Diffusion of sound in rooms - an overview. In Proceedings of the 15th International Congress on Acoustics, Trondheim, Norway, pages 633-636, 1995.

[22] J. H. Rindel. The use of computer modelling in room acoustics. Journal of Vibroengineering, 3(4):41-72, 2000.

[23] M. R. Schröder. New method for measuring reverberation time. Journal of the Acoustical Society of America, 37:409-412, 1965.

[24] M. R. Schröder. Diffuse sound reflection by maximum length sequences. Journal of the Acoustical Society of America, 57:149-151, 1975.

[25] M. H. A. Gomes; S. N. Y. Gerges; R. A. Tenenbaum. On the accuracy of the assessment of room acoustics parameters using mls technique and numerical simulation. Acustica united with Acta Acustica, 86(5):891, 2000.
[26] A. H. G. Rinnoykan; G. T. Timmer. Stochastic global optimization methods. Part I: Clustering methods. Mathematical Programming, 39:27-56, 1987.

[27] J. Vanderkooy. Aspects of MLS measuring systems. Journal of the Audio Engineering Society, 42(4):219-223, 1994.

[28] A. Lundeby; T. E. Vigran; H. Bietz; M. Vorländer. Uncertainties of measurements in room acoustics. Acustica, 81:344-355, 1995.

[29] M. Vorländer. Simulation of the transient and steadystate sound propagation in rooms using a new combined ray-tracing/image-source algorithm. Journal of the Acoustical Society of America, 86(1):172-178, 1989.

[30] M. Vorländer. International round robin on room acoustical computer simulations. In Proceedings of the International Conference on Acoustics 1995, Norway, 1995.

[31] O. Warusfel. Predictive acoustics software and computer aided optimization in room acoustics. In 15th International Congress on Acoustics, pages 693696, 1995. 